\title{
THE CONFORMAL TRANSFORMATION GROUP OF A COMPACT HOMOGENEOUS RIEMANNIAN MANIFOLD
}

\author{
BY SAMUEL I. GOLDBERG ${ }^{1}$ AND SHOSHICHI KOBAYASHI
}

Communicated by Raoul Bott, February 13, 1962

1. Introduction. Let $M$ be a connected Riemannian manifold of dimension $n, C_{0}(M)$ the largest connected group of conformal transformations of $M$ and $I_{0}(M)$ the largest connected group of isometries of $M$. In a previous paper [1], the authors have shown

A. A compact homogeneous Riemannian manifold for which $C_{0}(M)$ $\neq I_{0}(M)$ is a rational homology sphere.

The purpose of the present paper is to establish the following stronger statement:

THEOREM. Let $M$ be a compact homogeneous Riemannian manifold of dimension $n>3$. Then, if $C_{0}(M) \neq I_{0}(M), M$ is isometric with a sphere.

We list several results in the same direction.

B. Let $M$ be a complete Einstein space of dimension $n>2$. If $C_{0}(M)$ $\neq I_{0}(M)$, then $M$ is isometric with a sphere (Yano-Nagano [5]).

Making use of this fact, Nagano [4] proved

C. Let $M$ be a complete Riemannian manifold of dimension $n>2$ with parallel Ricci tensor. Then, if $C_{0}(M) \neq I_{0}(M), M$ is isometric with a sphere.

This generalizes (B).

Let $M$ be a Riemannian manifold with metric tensor $g$. A transformation $\phi$ of $M$ is said to be conformal if $\phi^{*} g=\rho g$ where $\rho$ is a positive function on $M$. If $\rho$ is a constant function, $\phi$ is called a homothetic transformation. If $\rho$ is identically equal to $1, \phi$ is an isometry.

2. Notation and formulae. Let $X=\xi^{i}\left(\partial / \partial x^{i}\right)$ be an infinitesimal transformation and $\xi=\xi_{i} d x^{i}$ the 1 -form associated with $X$ by means of the duality defined by the metric tensor $g=g_{i j} d x^{i} d x^{i}$, i.e., $\xi_{i}=g_{i j} \xi^{i}$ where repeated indices imply summation. Let $\theta(X)$ be the operator of Lie derivation with respect to $X$. An infinitesimal transformation $X$ is conformal, if and only if

$$
\theta(X) g=\lambda g
$$

1 The first named author was supported by the Air Force Office of Scientific Research. 
where $\lambda$ is a function on $M$. It is homothetic, if and only if $\lambda$ is a constant function, and it is isometric, if and only if $\lambda=0$. For a 1 -form $\xi$, we have

$$
\delta \xi=-\nabla_{i} \xi^{i}
$$

where $\delta$ is the co-differential operator and $\nabla_{i}$ denotes covariant derivation in the "direction" of $\partial / \partial x^{i}$. Hence, since

$$
\theta(X) g=\left(\nabla_{j} \xi_{i}+\nabla_{i} \xi_{j}\right) d x^{i} d x^{j},
$$

we obtain $-2 \delta \xi=n \lambda$. Thus, for an infinitesimal conformal transformation $X$,

$$
\theta(X) g=-\frac{2}{n} \delta \xi \cdot g .
$$

Consider a Riemannian manifold $M$ with metric $g$ and let $g^{*}$ be a conformally related locally flat metric (if it exists). Under the circumstances, $M$ is called (locally) conformally flat. As a consequence, the Weyl conformal curvature tensor $C$ with components

$$
\begin{gathered}
C_{j k h}^{i}=R_{j k h}^{i}-\frac{1}{n-2}\left(R_{j k} \delta_{h}^{i}-R_{j h} \delta_{k}^{i}+g_{j k} R_{h}^{i}-g_{j h} R_{k}^{i}\right) \\
+\frac{R}{(n-1)(n-2)}\left(g_{j k} \delta_{h}^{i}-g_{j h} \delta_{k}^{i}\right)
\end{gathered}
$$

(relative to the given system of local coordinates) vanishes where the first term on the right hand side denotes the components of the Riemannian curvature tensor, $R_{j k}=R^{i}{ }_{j k i}$ is the Ricci curvature, and $\delta_{k}^{i}$ is the Kronecker delta. Indeed, the tensor $C$ remains invariant under a conformal change of metric. Conversely, if $C=0$ and $n>3$, $M$ is conformally flat.

3. Proof of theorem. In the sequel, let $G=I_{0}(M)$ and $M=G / K$. The subgroup $K$ need not be connected.

Proposition 1. Let $M$ be a compact homogeneous Riemannian manifold. Then, if $C_{0}(M) \neq I_{0}(M)$, the fundamental group $\pi_{1}(M)$ of $M$ is finite.

Proof. Assume that $K$ is connected and consider the exact homotopy sequence

$$
\rightarrow \pi_{1}(K) \rightarrow \pi_{1}(G) \rightarrow \pi_{1}(M) \rightarrow 0 .
$$

Since $\pi_{1}(G)$ is abelian, so is $\pi_{1}(M)$. Hence 


$$
H_{1}(M, Z) \approx \pi_{1}(M) /\left[\pi_{1}(M), \pi_{1}(M)\right] \approx \pi_{1}(M) .
$$

On the other hand, by (A), the first Betti number of $M$ is zero. Consequently, $H_{1}(M, Z)$ is a finite group since it is a finitely generated torsion group. We may therefore conclude that $\pi_{1}(M)$ is a finite group.

If $K$ is not connected, let $K_{0}$ be the connected component of the identity in $K$ and consider the exact homotopy sequence.

$$
0 \rightarrow \pi_{1}\left(G / K_{0}\right) \rightarrow \pi_{1}(G / K) \rightarrow K / K_{0} \rightarrow 0 .
$$

Since $K$ is compact, $K / K_{0}$ is finite. Hence, since $\pi_{1}(G / K)$ is an extension of $\pi_{1}\left(G / K_{0}\right)$ by $K / K_{0}$, it is finite.

Proposition 2. The Riemannian manifold $M$ is conformally flat provided $n>3$ (cf. [6, p. 275]).

To see this, set $\langle C, C\rangle=g^{i p} g^{i q} g^{k r} g^{h s} C_{i j k h} C_{p q r s}$ where $C_{i j k h}=g_{i r} C^{r}{ }_{j k h}$. Then, with respect to an infinitesimal conformal transformation $X$,

$$
\begin{aligned}
\theta(X)\langle C, C\rangle & =\langle\theta(X) C, C\rangle+\frac{4}{n} \delta \xi\langle C, C\rangle \\
& =\frac{4}{n} \delta \xi\langle C, C\rangle .
\end{aligned}
$$

This is an immediate consequence of formula (1) and the fact that $\theta(X) C=0$. The manifold $M$ being homogeneous, and the tensor $C$ being invariant by $I_{0}(M),\langle C, C\rangle$ is a constant. Therefore, if $X$ is not an infinitesimal isometry, $\delta \xi \neq 0$, from which $\langle C, C\rangle=0$, that is $C$ must vanish. Hence, if $n>3, M$ is conformally flat.

Proposition 3. (KuIPER [2]). A compact, simply connected, conformally flat Riemannian manifold is conformal to an ordinary sphere.

Now, let $\tilde{M}$ be the universal covering space of $M$. Then, by Proposition $1, \tilde{M}$ is compact. Let $\pi$ be the projection of $\tilde{M}$ onto $M$. Then, the induced metric $\pi^{*} g$ is conformally flat. Applying Proposition 3, we see that $\tilde{M}$ is conformal to an ordinary sphere, that is, there exists a Riemannian metric $h$ on $\tilde{M}$ such that

(1) The Riemannian manifold $(\tilde{M}, h)$ is isometric with a sphere;

(2) $\pi^{*} g=\rho h$ where $\rho$ is a positive function on $\tilde{M}$.

Let $C_{0}(\tilde{M}, h)$ be the largest connected group of conformal transformations of $(\tilde{M}, h)$. Let $I_{0}(\tilde{M}, h)$ and $I_{0}\left(\tilde{M}, \pi^{*} g\right)$ be the largest connected groups of isometries of $(\tilde{M}, h)$ and $\left(\tilde{M}, \pi^{*} g\right)$ respectively. Since $\pi^{*} g$ and $h$ are conformally related, both $I_{0}(\tilde{M}, h)$ and $I_{0}\left(\tilde{M}, \pi^{*} g\right)$ are (compact) subgroups of $C_{0}(\tilde{M}, h)$. Since $I_{0}(\tilde{M}, h)$ is a maximal 
compact subgroup of $C_{0}(\tilde{M}, h)$, there exists an element $\phi$ of $C_{0}(\tilde{M}, h)$ such that

$$
I_{0}\left(\tilde{M}, \pi^{*} g\right) \subset \phi \cdot I_{0}(\tilde{M}, h) \cdot \phi^{-1} .
$$

Then the Riemannian metric $\phi^{*} h$ is invariant by $I_{0}\left(\tilde{M}, \pi^{*} g\right)$. Since both $\phi^{*} h$ and $\pi^{*} g$ are conformally related to the same metric $h$, there exists a positive function $\mu$ on $\tilde{M}$ such that

$$
\pi^{*} g=\mu \cdot \phi^{*} h \text {. }
$$

Since both $\phi^{*} h$ and $\pi^{*} g$ are invariant by $I_{0}\left(\tilde{M}, \pi^{*} g\right)$ which is transitive on $\tilde{M}, \mu$ is a constant function. Since $(\tilde{M}, h)$ is a space of constant curvature, so is $\left(\tilde{M}, \phi^{*} h\right)$. Hence, $\left(\tilde{M}, \pi^{*} g\right)$ is a space of constant curvature.

The manifold $M$ is consequently an Einstein space, and so, by (B), it is isometric with a sphere.

REMARK 1. The statement (B) is required only in the final step of the proof. Without it (but with the help of (A)) we may conclude that the universal covering space of a compact homogeneous Riemannian manifold, admitting a vector field generating a global 1parameter group of non-isometric conformal transformations, is isometric with a sphere.

REMARK 2 . In view of the results $B$ and $C$ and our theorem, we pose the following question. Let $M$ be a compact Riemannian manifold with constant (positive) scalar curvature such that $C_{0}(M) \neq I_{0}(M)$. Is $M$ isometric with a sphere? It is known that if $M$ is a compact Riemannian manifold of constant negative scalar curvature, then $C_{0}(M)=I_{0}(M)$ (see, for instance, $[3$, p. 134]).

\section{REFERENCES}

1. S. I. Goldberg and S. Kobayashi, The conformal transformation group of a compact Riemannian manifold, Proc. Nat. Acad. Sci. U.S.A. 48 (1962), 25-26; Amer. J. Math. 84 (1962), 170-174.

2. N. H. Kuiper, On conformally-flat spaces in the large, Ann. of Math. (2) 50 (1949), 916-924.

3. A. Lichnerowicz, Géométrie des groupes de transformations, Travaux et Recherches Mathématique, Dunod, Paris (1958).

4. T. Nagano, The conformal transformations on a space with parallel Ricci tensor, J. Math. Soc. Japan 11 (1959), 10-14.

5. K. Yano and T. Nagano, Einstein spaces admitting a one-parameter group of conformal transformations, Ann. of Math. (2) 69 (1959), 451-461.

6. K. Yano, The theory of Lie derivatives and its applications, North-Holland Publishing Co., Amsterdam, 1957.

UNIVERSITY OF ILLINOIS AND

University of British Columbia 